\title{
Adaptive UIX Layer for University Information SOA-BUS
}

\author{
Octavian DOSPINESCU, Cătălin STRÎMBEI, Roxana-Marina STRAINU, \\ Alexandra NISTOR \\ Faculty of Economics and Business Administration, AL.I.Cuza University, Iasi \\ doctav@uaic.ro,linus@uaic.ro,roxana.strainu@gmail.com, \\ alexandra.anichitoaei@yahoo.com
}

The user interface (UI) layer is considered one of the key components of software applications since it connects their end-users to functionalities. Well-engineered and robust software applications could eventually fail to be adopted due to a weak UI layer. In the current market, when creating sites, UI designers have to determine how to plan the best interface according to the devices of the users, namely desktops, laptops, tablets or smartphones. Having this in mind, the current paper aims to present the adaptive UIX Layer for a University Information SOA-Bus (UISB) - a topic discussed in previous works [1], [2]. In this respect, we reviewed the literature on responsive and adaptive web design in order to identify the best front-end approach to the UISB. According to our findings, there is a strong debate in the academic field as well in the IT business in what concerns UI design. Recent interests have shown that the new trend in drafting the front-end layer is choosing between a responsive and an adaptive design. While arguments provided by both sides have failed to ease the decision makers' choices on what type of design to choose, we aim to bring some light on the subject by proposing different layers for the UISB.

Keywords: SOA, User Interface, Responsive, Adaptive

$\mathbf{1}_{1}$ Introduction

The development of User Interfaces (UI) has been a topic of interest both for academia and industry since the early 1980s. During the time a constant evolution has occurred in the field and such an evolution has been coupled with a continuous effort to develop new methods and strategies to create UIs in effective and efficient ways [3].

UIX (User Interface XML) is a set of technologies that constitute a framework for building web applications. The main focus of UIX is the user presentation layer of an application, with additional functionality for managing events and the state of the application flow. UIX is designed to create applications with page-based navigation, such as an online human resources application, rather than full-featured applications requiring advanced interaction, such as an integrated development environment (IDE) [4].

An application can interact with UIX in predefined places called decision points, where a decision is made by the operator or a certain action routine is automatically triggered. Execution of an action terminates in a new decision point. The application's structure is provided to UIX in configuration files, which can be ASCII files, databases, or resource files. UIX includes Java class libraries, APIs, XML languages, and other technologies for developing different aspects of web-based applications [5].

\section{Responsive Web Design (RWD)}

Nowadays, responsive design has become a major trend in web development due to the high diversity of devices used for web browsing. According to [6] applying responsive design to existing web sites implies major reengineering due to the underlying fluid grid process. Likewise, responsive design is limited to desktop-tomobile adaptation.

The expression 'responsive web design' has earned popularity in 2010 when the web designer and developer Ethan Marcotte wrote an article on the subject [7]. The same author considers that the goal of RWD consists in making a web page look equally good no matter the screen size of a device. Before the introduction of responsive web design, web 
designers and developers created most websites by following the principles of pixelperfect web design. Pixel-perfect web design treats a web page like a page from a magazine. In this approach, the mock-up of a web page is first created in Photoshop, and then a developer recreates that design to fit a web browser. The goal of pixel-perfect web design is to make a web page resemble the original mock-up as much as possible. But a web page is not printed on a piece of paper but viewed in a web browser. Unlike paper, a web browser is a dynamic medium. It allows a user to re-size the browser window itself, and users can also change the size of the font as well. And when this happens, web pages created with pixel-perfect web design principles often break. If a web page was optimized for a 1024 $\times 768$ pixel screen size, for example, that web page will look quite wrong in a smaller or bigger screen [8].

As the number of mobile devices that have a variety of screen sizes grows, pixel-perfect web design has become problematic. Responsive web design is an attempt to solve this problem with the following three tools [9]:

- a flexible, grid-based layout;

- flexible images;

- media queries.

Flexible grids are created by using percentage (a relative unit) instead of pixel (an absolute unit). Media queries make it possible to apply different cascading style sheets (CSS) depending on the media type and the maximum width of the device screen. With cascading style sheets, one can control images and other fixed-width elements so that they stay contained in their container blocks.

Responsive web design makes a web page adjust itself in response to the screen size of a device. This means that there is no longer one fixed layout in which the elements of a web page are permanently placed. Instead, as the size of the screen changes, the layout of a web page adjusts itself and rearranges the elements of the page.

According to [10] and [11], responsive web design is used by many digital agencies due to its specific advantages, namely:
- The content stays the same - having this in mind, using RWD reduces the need to change or 'adapt' the interface website for different versions. Moreover, the Content Management System (CMS) remains unmodified, the need to duplicate content entry according to different screen types being eliminated.

- It can be easily achieved automatically - in order to determine the width of the browser or device there can be used CSS (cascading style sheets), media queries or HTML5, the existing content being adjusted or stacked to fit automatically the space available [11].

- Cost - in comparison with the Adaptable Web Design the Responsive one is cheaper and less time consuming.

Although the advantages shown make of RWD an attractive choice when designing interfaces, the same author recalls some limitations as:

- Generic not optimized experience when accessing a site with RWD interface, the user does not benefit of a fully optimized experience in accordance with the device they are using.

- No accounting for users' behavioral differences with different mediums. The evidence indicates that users behave very differently when using smartphones and desktops, so ideally a mobile website should be adapted to these behavioral changes [11].

- Loading time issues. Websites with lots of HTML code and multimedia take far longer to load on a mobile device with a $3 \mathrm{G}$ connection and a smaller CPU than on a desktop with broadband. As the content doesn't change between environments, with responsive websites, mobile users are kept waiting [11].

A responsive design can have multiple breakpoints, say for a small-screen phone, then a large-screen phone, then a tablet, then a laptop/desktop. Many teams try to decide on breakpoints using average screen sizes. However, it's better to look at what the content and navigation wants to be. By letting the content and navigation drive the 
breakpoints, teams find they can often get away with fewer screen configurations. For example, a high-resolution Retina iPad might easily share the same configuration as a wellconstructed laptop display, while lower resolution tablets might just need a little adjustment to that same configuration.

\section{Adaptive User Interfaces}

Adaptive User Interfaces (AUI) are particular context-aware systems that aim at generating user interfaces relevant to the user's profile, task and environment [12]. The AUI research domain comes from the crossover of the personalization domain (which consists in allowing the user to manually customize the interface) and the context-awareness. Hence, AUI focus on providing a user interface well suited to the user and its task with as less as possible user intervention. The main challenge for AUI is to design their mechanisms so that the usability is preserved [8].

Unlike standard context-aware systems, AUI promote a re-organization or a re-distribution of a user interface where context-aware systems associate a unique interface per service. On the detection of a new context, AUI may re-arrange the graphical components (and their properties such as the size), the available modalities or the type of visualization to better suit the new context. The quality of AUI depends on the relevancy of the adaptation considering the task and on the intrusiveness of the adaptation. An AUI that adapt too many times without the user's approbation may have a negative impact on the task.

AUI represent a complex problem addressed by particular approaches. A notable one is the "plasticity" approach which originally aimed at resolving interface adaptation problems considering an adaptation to the device (computing context) and to the physical environment (physical context) [13]. Plasticity employs a Model-Driven Engineering (MDE) tactic to design interface having multiple representations (one for each targeted context) and distributed interfaces (interfaces capable to display on several devices) [13]. Plasticity design suggests that the contexts are known at design time. The concept evolved to consider the user's context and more recent researches on plasticity introduced the consideration of time context by using learning systems to depict users' preferences and habits [14]. Plasticity focuses on modelling multi-target interfaces, distributed interfaces, physical environment, hardware and software capabilities and tasks. The mechanism of adaptation is commonly dealt through Service Oriented Architecture [12] or component based approach.

Another approach to context-aware interfaces is the USer Interface eXtensible Markup Language (UsiXML) (Limbourg, 2005). UsiXML is a user interface description language that aims at modeling relevant aspects of the interfaces to support contextaware application. For this purpose, UsiXML introduced the " $\mu 7$ " concept which consists in the design of "multi-device, multi-platform, multi-user, multi-linguality / culturality, multi-organization, multi-context and multimodality" enabled interfaces. UsiXML models are partly based on the works on plasticity and propose models to enable the adaptation of the interfaces to the user's context.

The commonly adopted alternative to RWD is AWD - Adaptive Web Design. According to [11], experts such as Jakob Nielsen have argued that AWD offers a preferable solution. The advantages are:

- Speed;

- Sensory design;

- Native app portability.

But AWD also has the following limitations:

- The content nightmare;

- Too many versions;

- Native app 'transformation'.

\section{Combining RWD with AWD}

The best solution is often achieved by combining RWD with AWD, in order to enjoy the cost and time efficiencies of RWD while offering the better user mobile experience that AWD offers in prioritized content areas. 
Table 1 summarizes the twelve principles that define AWD and RWD, comparing each method of use and implementation.

Table 1. Key principles in AWD and RWD [15]

\begin{tabular}{|c|c|c|}
\hline Principles & Adaptive Web Design (AWD) & $\begin{array}{c}\text { Responsive Web Design } \\
\text { (RWD) }\end{array}$ \\
\hline Access Speed & Very fast & Fast \\
\hline App Store & Available & Not necessary \\
\hline Approval Process & Some are mandatory & None \\
\hline Content Versions & $\begin{array}{l}\text { Multiple URLs/versions for each } \\
\text { page, i.e., content forking }\end{array}$ & $\begin{array}{l}\text { Same content regardless of } \\
\text { device or platform }\end{array}$ \\
\hline Development Cost & Expensive to very expensive & Moderate to reasonable \\
\hline Features & $\begin{array}{l}\text { Phone features, location services, } \\
\text { camera, etc... }\end{array}$ & Limited phone features \\
\hline Functionality & $\begin{array}{l}\text { Some functions may be omitted } \\
\text { from the mobile application }\end{array}$ & $\begin{array}{l}\text { All functions of original site } \\
\text { remain }\end{array}$ \\
\hline Internet Connection & Available offline & Required \\
\hline Monetization & Easily monetized & Not as easy to monetize \\
\hline Navigation & Interactive user interface & $\begin{array}{l}\text { Static but responsive user } \\
\text { interface }\end{array}$ \\
\hline $\begin{array}{l}\text { Optimized to mobile } \\
\text { device }\end{array}$ & Best & Good \\
\hline User Access & $\begin{array}{l}\text { After installation and some user } \\
\text { configuration/interaction }\end{array}$ & $\begin{array}{l}\text { Through browser with little to no } \\
\text { user configuration/interaction }\end{array}$ \\
\hline
\end{tabular}

According to [11], using RWD as the basis, there can be included some elements of AWD, changing some assets, content, or interfaces depending on whether the site is visited from a smartphone, tablet, or desktop-thus making a responsive website appear 'adaptive'. Similarly, in the AWD approach there can be embraced the RWD's responsiveness so the content and interfaces degrade gracefully on different size screens (RWD) until the 'break point' where a different version is applied (AWD).

This route poses two challenges: The interaction design/information architecture needs to be designed for both the desktop and mobile at the same time while considering the resizing, spatial distribution, and animation (if required) of elements on desktops, tablets, and mobile screens. Once this juncture has been crossed, the developers need to rely on all available technologies (media queries, browser sniffing, feature detection, and network speed analysis) to detect the multitude of resolutions and device capabilities while tailoring the end user experience to each particular device. This approach is bleeding edge and quite experimental.

As we can see from Table 1, each of the two design practices have different advantages and limitations. While the responsive design is client-side, meaning the whole page is delivered to the device browser (the client), and the browser then changes how the page appears in relation to the dimensions of the browser window, the adaptive design is server-side, meaning before the page is even delivered, the server (where the site is hosted) detects the attributes of the device, and loads a version of the site that is optimized for its dimensions and native features. Considering the pros and cons we propose further a frontend solution for the University Information SOA-Bus based on the AWD approach. 
5 Adaptive user interface model for University Information SOA-Bus (UISB)

As suggested by [16], a typical approach when developing adaptive interfaces consists in using a set of models to generate and further refine a user interface artefact until the final user interface is adapted to best match the user's need, given the constraints set by the context in which the user operates. In order to design the UI of the UISB we took into consideration the Cameleon reference framework [17] which comprises four different abstraction layers for adaptive interfaces (Figure 1).

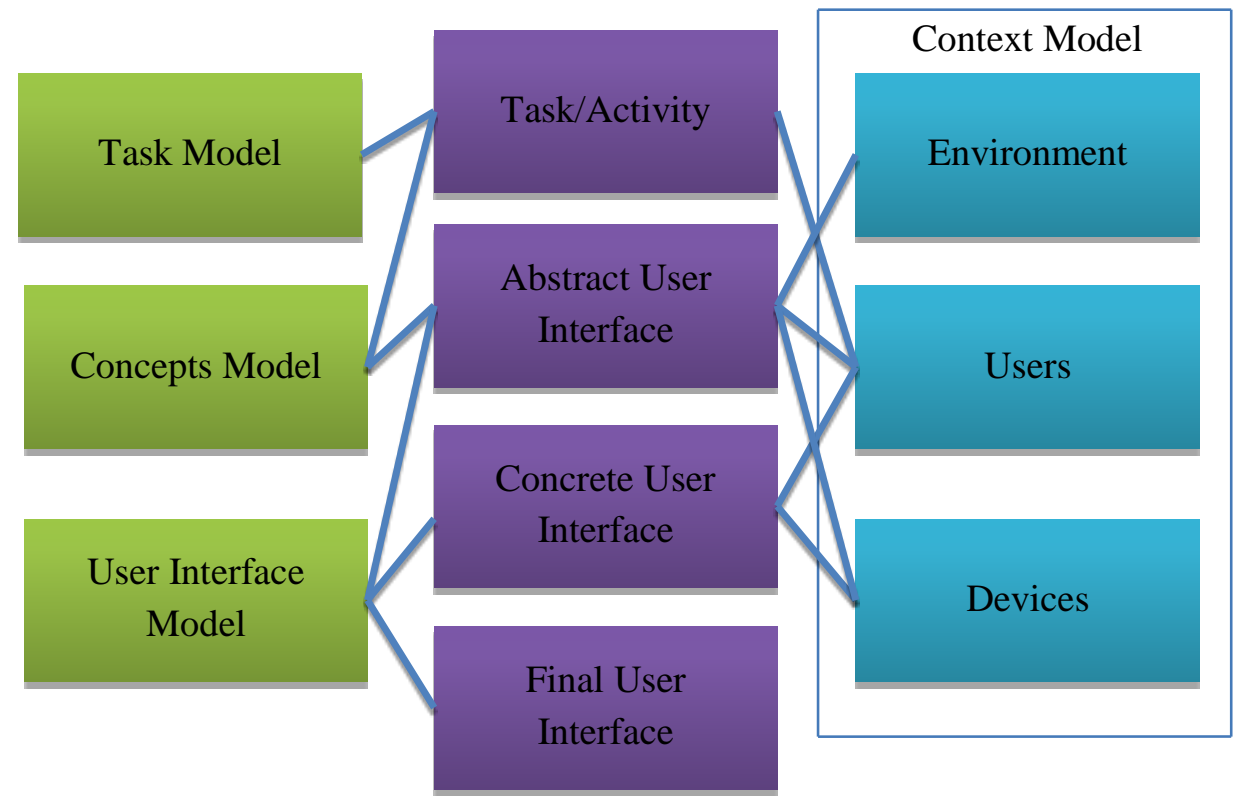

Fig. 1. Models and artifacts in the adaption process [4].

As shown in Figure 1, the interface adaption process uses four models, namely: task model, concept model, context model and user interface model. Further we present a short description for each of them.

Task model - when solving problems, the human being has the habit of decomposing it in several subproblems. In this regard, the most used structure for task models is the hierarchical one. In what concerns the UISB, we used the ConcurTaskTrees (CTC) proposed by [18] which contains four types of tasks:

$\checkmark$ System tasks, which are completed by the system alone;

$\checkmark$ User tasks, which are only allocated to a user;

$\checkmark$ Interaction tasks, which take place when the user relates to the system;

$\checkmark$ Abstract tasks.

Each of the four tasks can be further divided in subtasks, the connection between them being ensured by temporal operators. Using this notation, we can model the interactions and deal with the unexpected events which can occur in the University Information SOABus system (UISB). For example, if a professor, who is currently publishing a document on the Portal is required to evaluate a paper on the Blackboard, he will suspend the current activity and will solve the most urgent task suggested, the Blackboard evaluation.

Concepts model - according to [19], the concept model details the objects with which the user interacts through tasks. In the proposed UISB system we will use specific university concepts (e.g. admission, grades, evaluation, timetable, library).

Context model - stores relevant information collected and/or managed by the system [16]. In the UISB, the context model comprises the environment (which incorporates information regarding the environment in which the process executes), users (which holds information about the user's interest, goals and characteristics), and devices (in which are described the physical devices and software 
features of the platform the framework is running on).

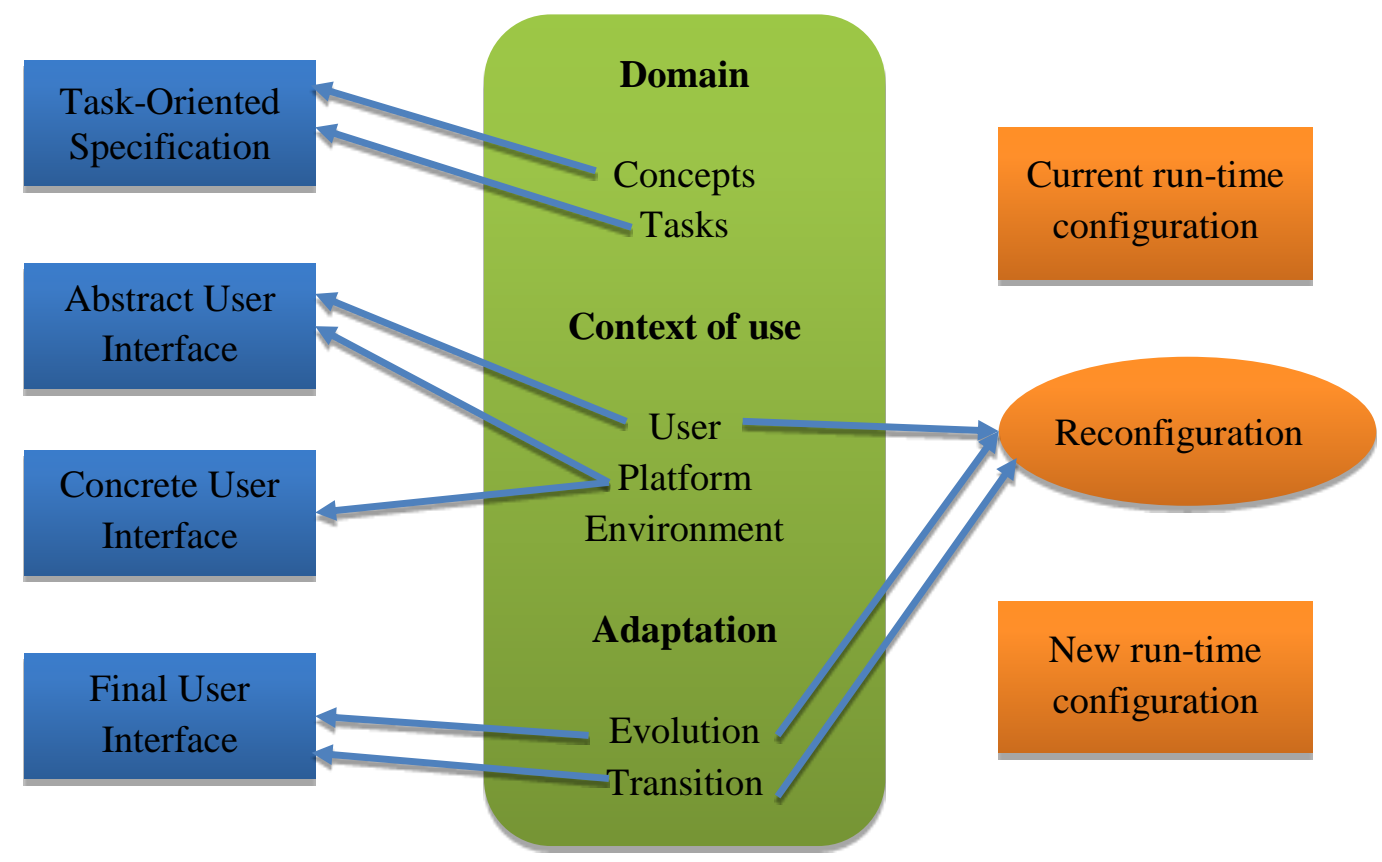

\section{Design phase:}

Developing different UI versions
Models used both in the design and run-time phase
2. Run-time phase: UI adjustment according to changes

Fig. 2. Phases in the adaption process [17]

User interface model - referred as the Final User Interface, is rendered by the UI toolkit of the given platform. In order to create an adaptive user interface for the UISB system, several UI versions will be developed in the design phase, while, at run-time, the proper changes will be made according to the adjustment required (see Figure 2).
Depending on the devices, desktop/mobile, the UI architecture differs in terms of structure. Further, we make a practical implementation of a web architecture for the proposed UISB.

The final goal is to obtain an adaptive user interface starting from the following class diagram. 


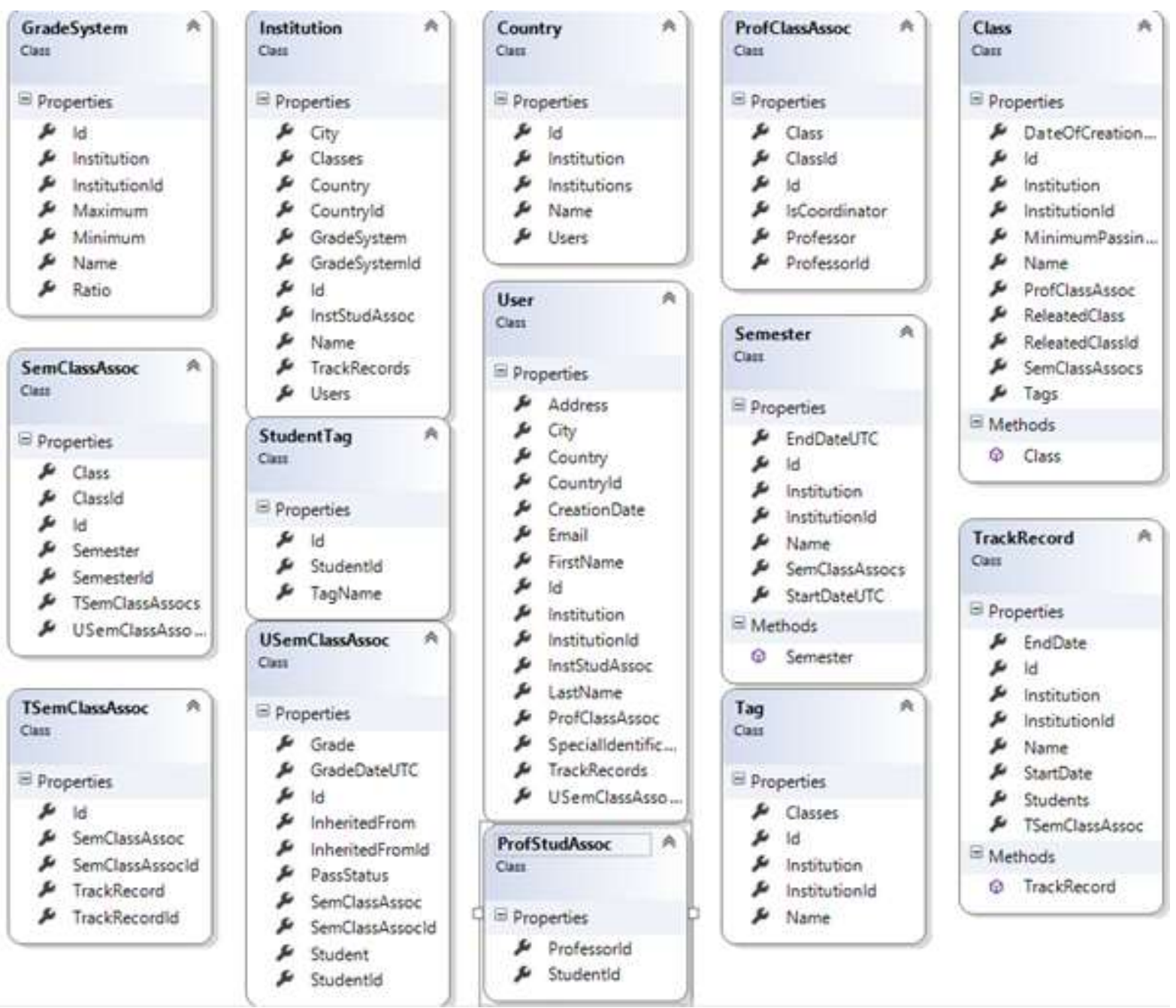

Fig. 3. The class diagram for the proposed system

The diagram contains the classes related to the education process, including information about students, classes, grades, track record, semesters and the associations between all these entities.

In order to implement the conceptual model, we used the .NET framework and its capabilities: entity framework model and model-view-controller technology. In the following sequences we present some pieces of representative code.

The model for StudentProfile is implemented as follows:

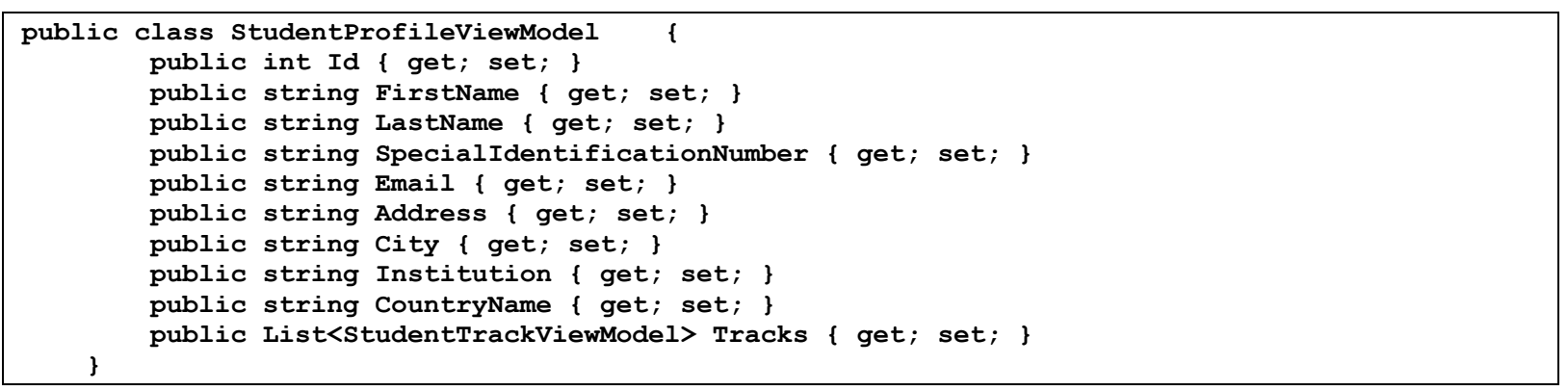

The controller implements the public method

GetProfile() which returns a result of IHttpActionResult type. 


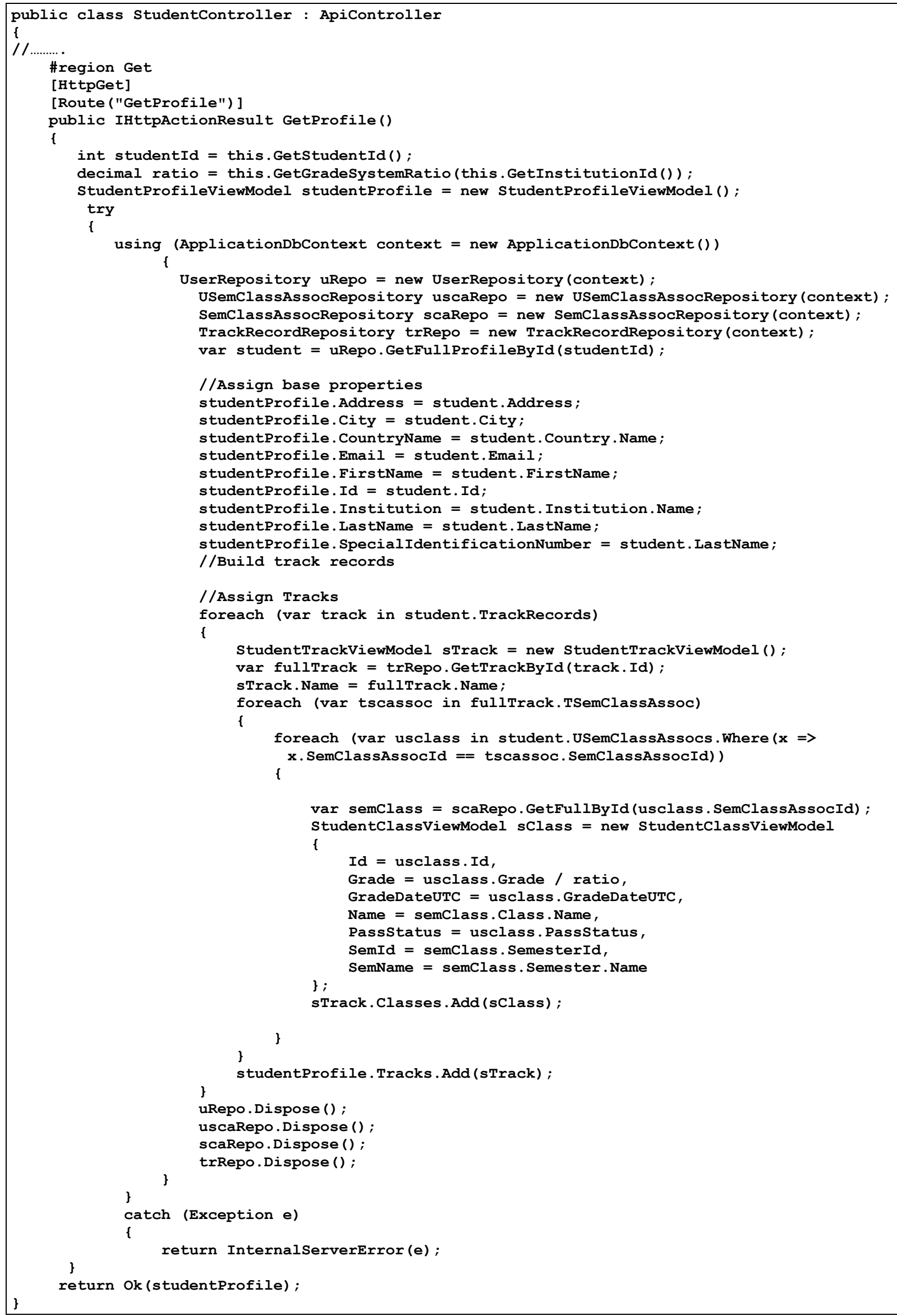


Based on the implemented models and the interface, according to the Figure 3 and Figure controllers we can get an adaptive user 4 .

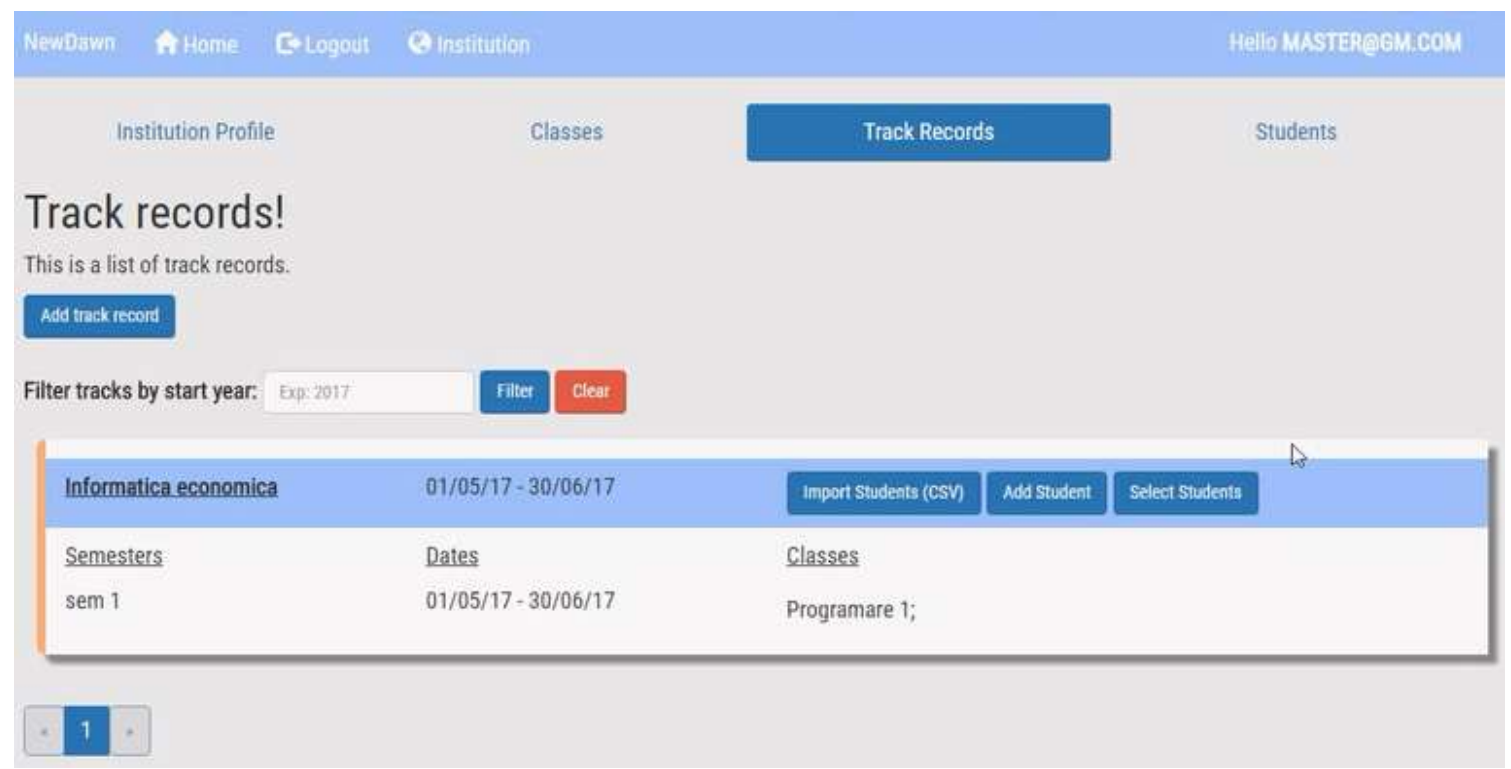

Fig. 3. The Adaptive User Interface for a specific track

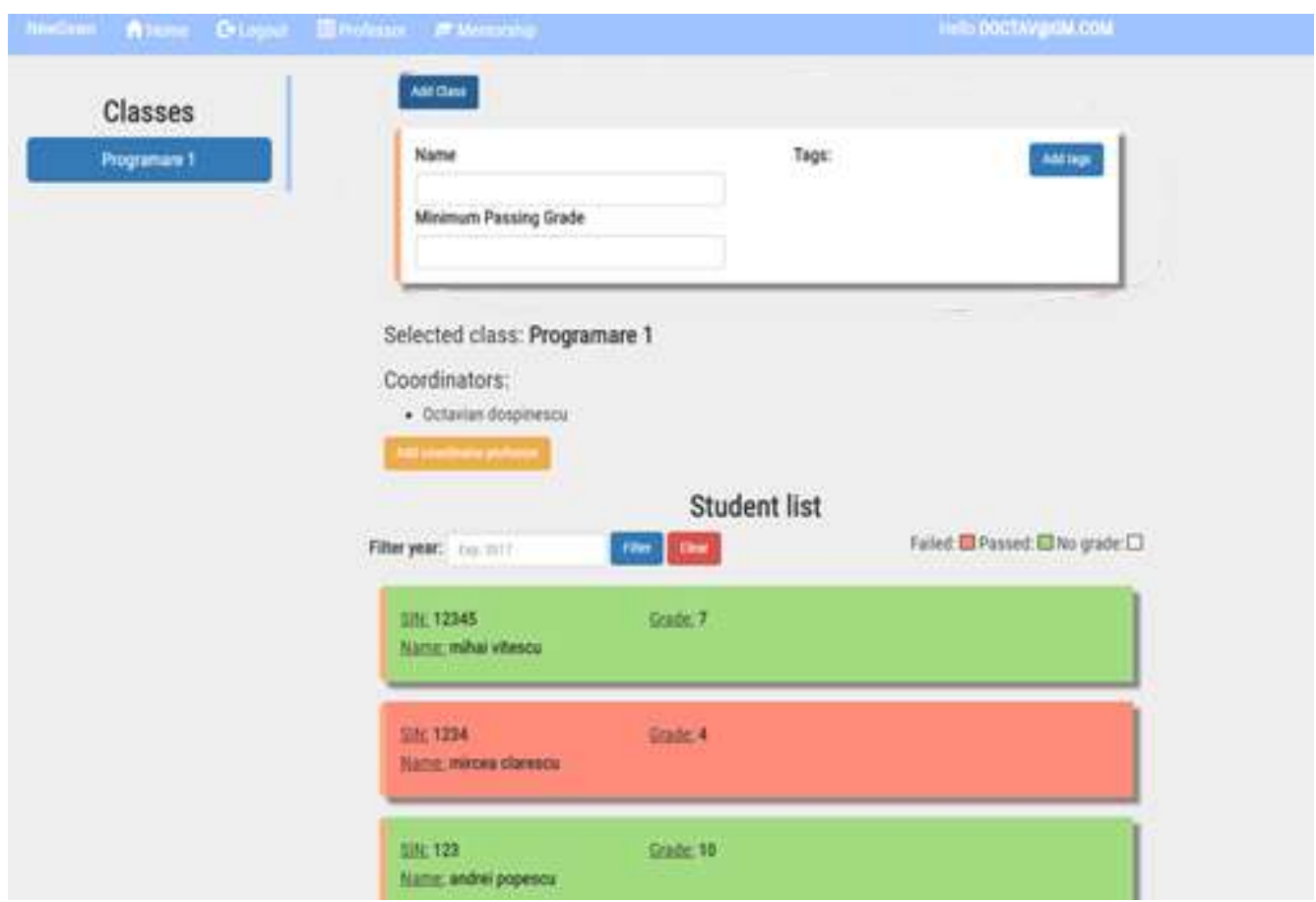

Fig. 4. The Adaptive User Interface for a specific class of students

The user interface uses the benefits of MVC (Model-View-Controller) paradigm and it gets the information offered by the controllers. The model is implemented in our prototype by using the entity-framework capability in .NET platform.

\section{Conclusion}

The need for a performant UISB has been addressed in several previous articles [1], [2]. After modelling the University Information Systems using a BPMN approach, and transforming the BPMN standard type services into a Service Oriented Architecture, in this article we aimed to develop a general 
model and a practical implementation on the .NET platform for the adaptive user interface (AUI) of the University Information SOABUS system (UISB).

The focus on the UI design revealed several trends among front-end developers. We refer mainly to the need to plan the best interface of an application according to the devices, the user's needs, the technologies used, the resources available to develop the system etc. In this regard, we noticed that there are two major designs used when creating the UI, namely responsive web design (RWD) and adaptive web design (AWD). While both of the design practices involve different requirements, the most used nowadays remains the Adaptive Web Design due to the specific optimization available according to the device from which the user access the application. Considering the wide spread of the AWD we proposed an adaptive user interface model for the UISB. Taking into account the Cameleon reference framework and the Model-View-Controller paradigm, we designed the models and artefacts in the adaption process, including the phases covered. As we have seen, there are different aspects that should be considered when designing the UI for different devices. More than that, the technologies used have a direct impact on the UI components and the way they are presented to the users.

\section{Acknowledgments:}

„This work was supported by a grant of the Romanian National Authority for Scientific Research and Innovation, CNCS UEFISCDI, project number PN-II-RU-TE2014-4-0748".

\section{References}

[1] C. Strîmbei, O. Dospinescu, R. Strainu and A. Nistor, "Software ArchitecturesPresent and Visions," Informatica Economica, vol. 19, no. 4, p. 13, 2015.

[2] C. Strîmbei, O. Dospinescu, R. Strainu and A. Nistor, "The BPMN Approach of the University Information Systems," Ecoforum Journal, vol. 5, no. 2, 2016.
[3] I. Zappia, D. Giuli, F. Paganelli and L. Chisci, Model and framework for multimodal and adaptive user interfaces generation in the context of business processes development, Firenze: Universita degli Studi Firenze, 2014.

[4] J. Vogt and A. Meier, "An adaptive user interface framework for eHealth services based on UIML," in Proceedings of the 23rd Bled eConference eTrust: Implications for the Individual, Enterprises and Society, Bled, 2010.

[5] S. Bongartz, Y. Jin, F. Paternò, J. Rett, C. Santoro and L. Spano, "Adaptive user interfaces for smart environments with the support of model-based languages," in International Joint Conference on Ambient Intelligence, 2012.

[6] M. Nebeling and M. Norrie, "Responsive design and development: methods, technologies and current issues," in International Conference on Web Engineering, 2013.

[7] E. Marcotte, "Responsive Web Design," 5 2010. [Online]. Available: https://alistapart.com/article/responsiveweb-design. [Accessed 0902 2017].

[8] T. Altenburger, A. Guerriero, A. Vagner and B. Martin, "Toward adaptive contextaware user interfaces for better usability and productivity in aec collaborative tasks," in Proceedings of the CIB W78 2010: 27th International Conference, 16-18 November, Egypt, Cairo, 2010.

[9] S. Mohorovičić, "Implementing responsive web design for enhanced web presence," in Information \& Communication Technology Electronics \& Microelectronics (MIPRO), 2013.

[10] B. Gardner, "Responsive web design: Enriching the user experience." Sigma Journal: Inside the Digital Ecosystem, vol. 11, no. 1, pp. 13-19, 2011.

[11] D. Bluestone, "Combining Responsive and Adaptive Strategies to Solve Mobile Design Challenges," 210 2012. [Online]. Available:

http://uxmag.com/articles/combiningresponsive-and-adaptive-strategies-tosolve-mobile-design- 
challenges?rate $=\mathrm{vXJQiJlBnkK7}$ _u2h-iikZIuNOxmXlqtEERci7KF0oQ.

[Accessed 183 2017].

[12] J. Coutaz, J. Crowley, S. Dobson and D. Garlan, "Context is the key," Communications of the ACM, vol. 48, no. 3, pp. 49-53, 2005.

[13] G. Calvary, J. Coutaz, D. Thevenin, Q. Limbourg, L. Bouillon and J. Vanderdonckt, "A unifying reference framework for multi-target user interfaces," Interacting with computers, vol. 15, no. 3, pp. 289-308, 2003.

[14] V. Ganneau, G. Calvary and R. Demumieux, "Learning Key Contexts of Use in the Wild for Driving Plastic User Interfaces Engineering," in Engineering Interactive Systems 2008 (2nd Conference on Human-Centred Software Engineering (HCSE 2008) and 7th International workshop on TAsk MOdels and DIAgrams (TAMODIA 2008, Pisa, Italy, 2008.

[15] K. Gajos, K. Everitt, D. Tan, M. Czerwinski and D. Weld, "Predictability and accuracy in adaptive user interfaces," in Proceedings of the SIGCHI Conference on Human Factors in Computing Systems, 2008.

[16] V. Jaquero, F. Montero, J. Molina and P. Gonzalez, "Intelligent User Interfaces: Past, Present and Future," Engineering the User Interface, pp. 1-12, 2009.

[17] L. Balme, A. Demeure, N. Barralon, J. Coutaz and G. Calvary, "Cameleon-rt: A software architecture reference model for distributed, migratable, and plastic user interfaces." in European Symposium on Ambient Intelligence, 2004.

[18] F. Paternò, C. Santoro and L. Spano, "ConcurTaskTrees," HIIS Laboratory, ISTI-C.N.R., Pisa, Italy, 2010.

[19] J. Van den Bergh and K. Coninx, "Model-based design of context-sensitive interactive applications: a discussion of notations," in TAMODIA '04 Proceedings of the 3rd annual conference on Task models and diagrams, Prague, Czech Republic, 2004.

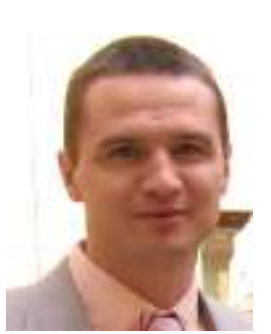

Octavian DOSPINESCU graduated the Faculty of Economics and Business Administration in 2000 and the Faculty of Informatics in 2001. He achieved the $\mathrm{PhD}$ in 2009 and he has published as author or co-author over 30 articles. $\mathrm{He}$ is author and co-author of 10 books and teaches as an associate professor in the Department of Information Systems of the Faculty of Economics and Business Administration, University Alexandru Ioan Cuza, Iasi. Since 2010 he has been a Microsoft Certified Professional, Dynamics Navision, Trade \& Inventory Module. In 2014 he successfully completed the course "Programming Mobile Applications for Android Handheld Systems" authorized by Maryland University. He is interested in mobile devices software, computer programming and decision support systems.

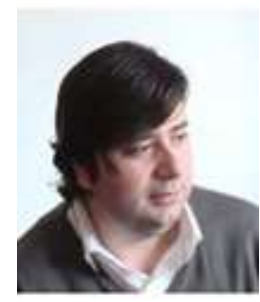

Cătălin STRÎMBEI has graduated the Faculty of Economics and Business Administration of Al.I.Cuza University of Iaşi in 1997. He holds a $\mathrm{PhD}$ diploma in Cybernetics, Statistics and Business Informatics from 2006 and he has joined the staff of the Faculty of Economics and Business Administration as teaching assistant in 1998 and as associate professor in 2013. Currently he is teaching Object Oriented Programming, Multi-Tier Software Application Development and Database Design and Administration within the Department of Business Information Systems, Faculty of Economics and Business Administration, Al.I.Cuza University of Iaşi. He is the author and co-author of four books and over 30 journal articles in the field of object oriented development of business applications, databases and object oriented software engineering. 


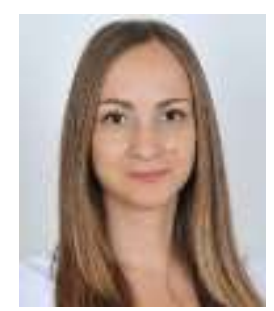

Roxana-Marina STRAINU graduated in 2014 the Master of Business Information Systems at the Faculty of Economics and Business Administration, Alexandru Ioan Cuza University of Iasi. She also graduated the Faculty of Mathematics in the year 2005. She is interested in developing smart systems and mobile applications on Android platform. Now she is a PhD student in the business information systems area.

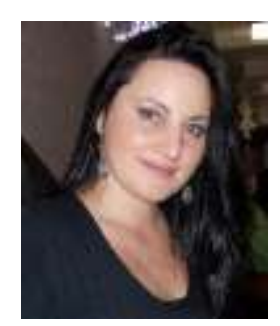

Alexandra NISTOR graduated the Faculty of Economics and Business Administration in 2011 and the Master of Business Information Systems at the Faculty of Economics and Business Administration in 2013. Her research interests include the use of automated testing in small and medium companies. Now she is a PhD student in the business information systems area. 